\title{
The Quantum Hall Effect
}

\author{
R.H. Morf \\ Paul Scherrer Institut \\ Zürich, Switzerland
}

More than 10 years after its discovery by $\mathrm{K}$. von Klitzing, G. Dorda and M. Pepper [1] the quantum Hall effect has remained a topic of intense and very fruitful research for both experimentalists and theorists. The field is rich in unexpected discoveries, some of them still unexplained. In this article, we shall first give a brief overview of the basic facts and then go on to discuss some of the more recent findings.

The quantum Hall effect occurs in artificial structures, e.g. silicon field effect transistors or semiconductor heterostructures, in which electrons are trapped at the interface between a large band-gap material and a smaller band-gap semiconductor in very thin layers. These electrons form two-dimensional systems and are essentially free to move in a plane parallel to the interface, while movement in the perpendicular direction is virtually impossible.

The Hall effect is observed by placing such a system in a perpendicular magnetic field $B$ and then passing a current $I_{x}$. The magnetic field deflects the moving electrons through the action of the Lorentz force in a direction perpendicular to the field and the direction of motion. This leads to an accumulation of electrons on one edge of the sample and a deficit of electrons at the opposite edge, thus creating a voltage difference in the $y$-direction, the Hall voltage $V_{H}$ across the sample, perpendicular to the current flow. The Hall resistance $R_{H}$ is defined as the ratio $R_{\mathrm{H}}=V_{\mathrm{H}} / I_{\mathrm{x}}$ and is proportional to the applied magnetic field $B$ and inversely proportional to the density $n_{\mathrm{S}}$ of current carrying electrons,

$$
R_{\mathrm{H}}=-B / n_{\mathrm{S}} e c
$$
where $c$ is the speed of light. The ordinary resistance $R_{\mathrm{x}}$ defined by the ratio $R_{\mathrm{x}}=$ $V_{x} / I_{x}$ of the voltage drop $V_{x}$ along the direction of the current and the current $I_{x}$ usually depends only weakly on the applied magnetic field.

By placing such a device in a very large magnetic field, and by studying it at very low temperatures, von Klitzing made the amazing discovery that the Hall voltage does not increase smoothly with increa-

Rudolf Morf is a theoretical physicist at the Paul Scherrer Institut, $\mathrm{CH}-8048$ Zürich. $\mathrm{He}$ studied at Basle University, receiving a PhD in nuclear physics in 1972. After spending a year at IBM Research, Rüschlikon, he was a postdoc at Harvard University before joining the RCA Laboratory (presently PSI). sing magnetic field, but exhibits plateaux in which the Hall voltage stays constant. And even more suprisingly, the values of the inverse of the Hall resistance $R_{H}{ }^{-1}$ at these plateaux are quantized in units of $e^{2} / h$,

$$
R_{\mathrm{H}}^{-1}=i\left(e^{2} / \mathrm{h}\right)
$$

where $i$ is an integer $i=1,2,3, \ldots$ Since equation (1) is generally valid, this result implies that the density $n_{\mathrm{S}}$ of current carrying electrons must have a very peculiar behaviour as a function of the magnetic field. In the range of the plateaux, simultaneously, the ordinary, longitudinal resistivity $\sigma_{x x}$ shows pronounced minima, at which it drops to exponentially small values at low temperature, implying dissipation-free charge transport at zero-temperature (see Fig. 1). The fact that in nonideal, "dirty" semiconductor devices, as found in modern electronics, an experiment could be carried out which would reveal with unprecedented accuracy the values of the fundamental constants of electromagnetism and quantum mechanics, namely the combination $e^{2} / h$, came as a surprise.

It was soon recognized that the quantization of the Hall resistance and, its insensitivity to the geometry and detailed structure of the device, had to be a consequence of a fundamental principle. R.B. Laughlin was the first to realize that gauge invariance could be used to derive the quantization of the Hall conductance, under the condition that an energy gap exists in the spectrum of extended electron states [2, 3]. Dissipation-free transport at zero temperature results for values of the Fermi energy in the range of the gap between extended states. This also implies a connection between the width of the Hall plateau and the size of the mobility gap.

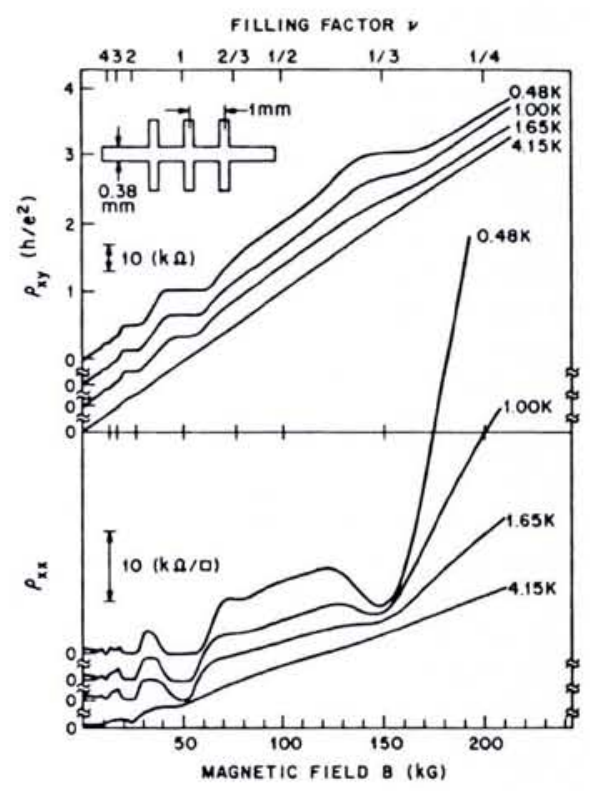

Fig. 1 - Longitudinal $\rho_{x x}$ and Hall $\rho_{x y}$ resistivities versus the magnetic field $B$ for a GaAs-Al ${ }_{0.3} \mathrm{Ga}_{0.7}$ As heterostructure sample, with $n_{S}=1.23 \times 10^{11} / \mathrm{cm}^{2}$, mobility $\mu=$ $90000 \mathrm{~cm}^{2} / \mathrm{Vs}$, using a current $\mathrm{I}_{\mathrm{x}}=1 \mathrm{~A}$. The Landau level filling factor is defined by $v=n h / e B$. (From [4], with permission.)

\section{Fractional Quantum Hall Effect}

Shortly after the discovery of the quantized Hall effect, Tsui, Störmer and Gossard [4] reported the observation of additional Hall plateaux at intermediate values, corresponding to fractional values of the integer $i$ in equation (2) above, namely $i=1 / 3,2 / 3$, etc. This phenome-

Table 1 - Energy and length scales for two dimensional electrons in a magnetic field $B$ (units of tesla). Parameters for $\mathrm{GaAs}-\mathrm{Al}_{\mathrm{x}} \mathrm{Ga}_{1-x} \mathrm{As}$ heterostructures of effective electron mass $m^{*}=0.07 m_{0}, g=0.4$ and dielectric constant $\varepsilon=12.8$ are used.

\begin{tabular}{|c|c|c|}
\hline Magnetic length & $I_{0}=(\hbar c / e B)^{1 / 2}$ & $\approx 26 \mathrm{~nm} \times B^{-1 / 2}$ \\
\hline Area per flux unit & $\Phi_{0} / B=2 \pi I_{0}^{2}$ & $\approx 4 \times 10^{11} \mathrm{~cm}^{2} / B$ \\
\hline Filling fraction & $v=n_{s} 2 \pi I_{0}^{2}$ & $=n_{s} \Phi_{0} / B$ \\
\hline Cyclotron energy & $E_{c}=\hbar \omega_{c}$ & $\approx 190 \mathrm{~K} \times B$ \\
\hline Zeeman energy & $E_{z}=g \mu_{B} B$ & $\approx 0.3^{\circ} \mathrm{K} \times \mathrm{B}$ \\
\hline Coulomb interaction & $V_{c}=e^{2} / \varepsilon l_{0}$ & $\approx 50^{\circ} \mathrm{K} \times B^{1 / 2}$ \\
\hline Subband splitting & $E_{s}$ & $\approx 200-500^{\circ} \mathrm{K}$ \\
\hline
\end{tabular}


non is now known as the fractional quantum Hall effect (FQHE). In contrast to von Klitzing who had employed silicon MOS devices, this group worked with a high quality $\mathrm{GaAs}-\mathrm{Al}_{\mathrm{x}} \mathrm{Ga}_{1-x}$ As heterostructure. Indeed, the stabilization of the system at electron densities corresponding to a fractionally filled Landau level requires electron interaction effects which can manifest themselves only in systems with sufficiently high electron mobility.

\section{Laughlin's FQHE Theory}

As a starting point for discussing Laughlin's theory of the FQHE effect, it is useful to recall the relevant distance and energy scales for the two-dimensional electron system in a perpendicular magnetic field $B$. Table 1 lists typical values for electrons trapped at the interface in a $\mathrm{GaAs}-\mathrm{Al}_{\mathrm{x}} \mathrm{Ga}_{1-x} \mathrm{As}$ heterostructure. On the basis of these energy scales, it becomes clear that for sufficiently large magnetic fields, the effects of Landau level mixing due to the Coulomb interaction will become less important, and that systems will be spin polarized.

In a seminal paper, Laughlin [5] proposed a trial state to describe the electron system at filling fraction $v=1 / m$,

$$
\begin{aligned}
& \psi_{\mathrm{m}}\left(z_{1}, \ldots, z_{N}\right) \\
& =\prod_{1 \leq i \leq j \leq N}\left(z_{i}-z_{j}\right)^{m} \prod_{k=1}^{N} \exp \left(-\left|z_{k}\right|^{2} / 4 I_{0}^{2}\right)
\end{aligned}
$$

This so-called Laughlin-Jastrow (L-J) wavefunction may be regarded as a Jastrow function built entirely from states of the lowest Landau level, which in the symmetric gauge are polynomials in $z=x+i y$ times the Gaussian $\exp \left(-\left|z_{k}\right|^{2} / 4 /{ }_{0}^{2}\right)$. The wavefunction is unique up to its exponent $m$, which must be an odd integer to satisfy the Pauli principle. The probability density $\left|\psi_{m}\right|^{2}$ is identical with that of the two-dimensional one component plasma at a suitably chosen coupling strength and represents a circular drop of fluid for not too large $m<70$. Its density is fixed by the positive background term (Gaussian part of the wave function) at $n_{\mathrm{S}}$ $=1 / 2 \pi m l_{0}^{2}$, from which the filling fraction $v=1 / m$ follows. For $m=v=1$, the Laughlin state coincides with the state of a filled Landau level.

The remarkable property of this state lies in the fact that while quite generally a state at filling fraction $v<1$ is constructed from $O(N / v)$ states and has $O(N / v)$ zeros, these zeros are actually all located at positions of electrons so large contributions to the Coulomb energy at short separation are suppressed. Indeed, for $m$. $=3, v=1 / 3$, this wavefunction leads to a pair correlation function which vanishes as the 6th power of the pair separation, while for any $v>1 / 3$ it must vanish as the square of separation [6]. Trial states with one more(less) zero in each electron coordinate were also constructed by Laughlin.

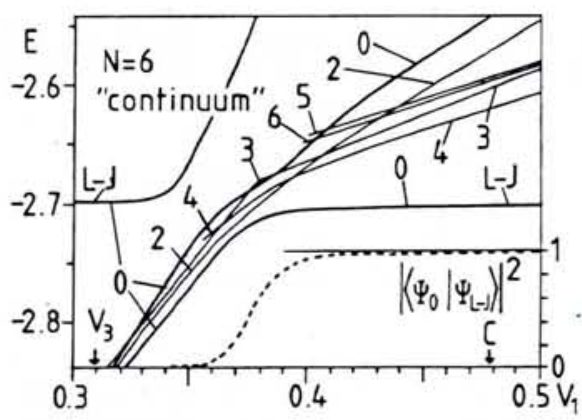

Fig. 2 - Low-lying states at $N=6,2 S=15$ $(v=1 / 3)$ as the "hard core" pseudopotential component $V_{1}$ is varied, where $2 S$ is the number of flux quanta $\mathrm{h} / \mathrm{l}$. The other $V_{m}$ take their Coulomb values. $V_{3}$ and the Coulomb value (C) of $V_{1}$ are indicated. Angular momentum quantum numbers $L$ are indicated. Also shown is the projection of the Laughlin-Jastrow $(L-J)$ state on the ground state. (From [7], with permission.)

They are obtained from by multiplication with $\Pi z_{\mathrm{i}}$ or by differentiating its polynomial part by $\Pi\left(\partial / \partial z_{i}\right)$. Again by the plasma argument, Laughlin was able to show that these states exhibit at the origin missing or excess charge $\pm \mathrm{e} / \mathrm{m}$, and thus represent fractionally charged excitations. Both these charged quasiparticles require a finite creation energy $\varepsilon^{+}$and $\varepsilon^{-}$ and lead to a gap $E_{\mathrm{g}}=\varepsilon^{+}+\varepsilon^{-}$in the excitation spectrum. The system in the Laughlin state has thus the characteristics of an incompressible fluid. Provided that the Laughlin state is very close to the actual ground state of the system, the conditions for the occurrence of a quantized Hall plateau at $v=1 / m$ are therefore met.

\section{Experimental Verification}

A very convincing test of Laughlin's trial state was performed by Haldane and Rezayi [7] who studied the exact ground state at $v=1 / 3$ for varying interaction. Note that within a Landau level, any pair interaction is fully specified by its expectation values $V_{L}$ in two-electron states of given relative angular momentum $L$. Fig. 2 shows the results obtained by varying $V_{1}$, keeping all other $V_{L}$ at their Coulomb values. Note the large overlap between the exact ground state and the Laughlin state for $V_{1}>0.4$ and its rapid decrease to zero below 0.4 , while at the same time the gap between the ground state and the first excited state disappears.

An experimental test was carried out by Willett et al. [8], in a study of the gap in a very high-mobility heterostructure, who measured the activation energy of the longitudinal resistivity $\rho_{x x}=\exp \left(-E_{\mathrm{g}} / 2 T\right)$ at $v=1 / 3,2 / 3,4 / 3$, and $5 / 3$. Their conclusion: the experimental gaps are of the order a factor of 2 smaller than theoretical results based on the pure Coulomb interaction. Taking into account the finite width of the wavefunction in the direction perpendicular to the interface and corrections due to Landau level mixing essentially eliminates the discrepancy, for sufficiently large magnetic fields. For small magnetic fields, there appears to be a threshold field below which no gap is observed: it may well result from the effects of impurities.

\section{Higher Order Fractional States}

With the development of improved samples of very high mobility, due in particular to the technique of modulation doping in which the donor ions are separated from the $2 \mathrm{~d}$ electron system, more Hall plateaus appeared at filling fractions, e.g., $v=2 / 5,3 / 7,2 / 7$, etc. These clearly could not be explained on the basis of Laughlin's wavefunction alone. A hierarchical theory was conceived by Halperin [9] and by Haldane [6]. Their theories are based on the idea that the fractionally charged excitations (quasiparticles), which are present in the Laughlin state if the electron density deviates from its precise value at, e.g. $v=1 / 3$, could themselves condense into an incompressible fluid, similar to that formed by the electrons in the Laughlin state. This would occur at particular densities of the quasiparticles. In this connection, Halperin [9] realized that a natural description of these quasiparticles required the introduction of fractional statistics, the quasiparticles in the quantum Hall system behaving as "anyons". As a consequence, allowed relative angular momenta have fractional values, $L=2 k \pm 1 / m$, for pairs of quasiholes or quasielectrons, respectively.

Both these hierarchical theories predict the occurence, in principle, of fractional plateaux for all rational filling fractions $0<v<1$ with an odd denominator. Indeed, treating the quasiparticles as point charges, and making a crude approximation for their interaction energy, Halperin obtained a ground state energy which, as a function of filling fraction, is everywhere continuous, but nowhere differentiable [9]. Naturally, at very low electron (or quasiparticle) densities the classical Wigner crystal (of electrons or quasiparticles) must be expected to have a lower energy than the Laughlin state: for corresponding densities, no quantum Hall plateau will occur.

The hierarchical theory makes precise predictions for the quantum numbers of the ground and excited states in finite systems. These have been verified in finite system studies by Haldane and Rezayi [7] and by d'Ambrumenil and myself [10].

However, the hierarchy seems to predict the existence of $\mathrm{FOH}$ states which do not occur in experiments. While, experimentally, the most stable fractional states occur along the sequence $v=2 / 5,3 / 7$, $4 / 9, \ldots, n /(2 n+1)$, this does not seem to follow naturally from the hierarchical scheme and needs clarification. In colla- 
boration with Pierre Béran, the author has undertaken an investigation of the quasiparticle interaction: it is found to differ strongly from the Coulomb interaction of point particles, in particular at short separation. As a result, the stability of quasiparticle Laughlin states which lead to $\mathrm{v}=$ $2 / 5,3 / 7,4 / 9$ is strongly enhanced, while other daughter states of $v=1 / 3$, e.g. $v=$ $4 / 11$, corresponding to lower density of quasiparticles are destabilized and gapless [11].

\section{Fractionally Charged Excitations}

A comprehensive experiment by Clark et al. [12] has investigated the longitudinal resistivity $\rho_{x x}$ of different fractional quantum Hall states at filling fractions $v=$ $p / q$, with $q=3,5,7$ and 9 . The authors analyzed the temperature $T$ dependence of $\rho_{x x}$ in the middle of a Hall plateau. Fitting the data to a single exponential

$$
\rho_{x x}(\beta)=\rho_{x x}^{c} \exp (-\Delta / T) \text {, }
$$

the prefactor $\rho_{x x}^{c}$ which results from extrapolation to $T=\infty$, could be used to determine an extrapolated value of the longitudinal conductivity

$$
\sigma_{x x}^{c}=\rho_{x x}^{c} /\left\{\left(\rho_{x x}^{c}\right)^{2}+\rho_{x y}{ }^{2}\right\} .
$$

Using for $\rho_{x y}$ its value at the plateau, $\rho_{x y}$ $=h / e^{2} v$, they found that all their experimental results could be fitted to the relation, $\quad \sigma_{x x}^{c}=c^{\prime}(e / q)^{2} / h$

in terms of the fractional charge $e^{*}=e / q$, and with a unique constant $c^{\prime}$, found to be close to unity. These results may indicate that quasiparticle excitations do indeed have fractional charge $e / q$, determined solely by the denominator, consistent with Laughlin's theory and with its extensions by Haldane [6] and by Halperin [9].

\section{The Role of Spin}

We have assumed so far that all the fractional quantum Hall states are fully polarized. In fact, the small magnitude of the Zeeman energy, see Table 1, was pointed out by Halperin in 1983. He proposed trial wavefunctions of the Laughlin type for systems in which both spins are populated. At $v=2 / 5$, a very natural wavefunction can be constructed, by allocating in equation (3) two different exponents $m$ for pairs of electrons with like spin and $n$ for pairs with unlike spin, yielding a filling fraction $v=2 /(m+n)$ where symmetry requires $m$ odd and $n$ even. Eisenstein et al. [13] recently observed a phase transition between an unpolarized state for small magnetic fields to a polarized state at large magnetic fields for $v=8 / 5$, which is the particle-hole conjugate of $v=2 / 5$, as suggested by Halperin. This phase transition, driven by the Zeeman energy by addition of an in-plane component of the magnetic field which does not change the filling factor, is accompanied by a gap which varies in an anomalous way with applied field. Other examples at various filling fractions have been investigated by Clark et al. [14].

\section{Even Denominator FQH State}

A fractional quantum Hall state at a Landau level filling fraction $v=5 / 2$ has been detected recently [15]. It is the first even denominator fraction for which not only minima in the longitudinal resistivity $\rho_{x x}$ but also a plateau at a Hall conductance $\sigma_{x y}=v e^{2} / h$ has been observed. The experiment was carried out with an extremely high electron mobility sample. Further experimental studies by the same group [15] have shown that the state disappears rather suddenly if the magnetic field is tilted away from the direction perpendicular to the semiconductor interface, by adding a field component $B_{1}$ parallel to the interface and keeping the perpendicular component $B_{0}$ constant. This tilting allows the Zeeman splitting to increase without affecting the filling fraction $v$. The sudden disappearance of the Hall plateau for increasing $B_{1}$ has been taken as evidence that a small Zeeman splitting is crucial for this state, which thus appears as though it is either unpolarized or maybe only slightly polarized.

An elegant suggestion for a possible trial wavefunction for this even denominator $\mathrm{FQH}$ state has been proposed by Haldane and Rezayi [16] in which electrons with opposite spin are grouped into tightly bound pairs. In the same way as the Laughlin wave function at $1 / m$ is the exact ground state for a type of hard-core repulsive interaction, the even denominator state is the exact ground state for a suitable model interaction which favours unpolarized over fully polarized states, and which has zero energy in pair states with zero relative angular momentum, thereby favouring tight pairing. However, this state appears not to be the ground state, even if the interaction matrix elements for the $n=1$ Landau level are employed. Other models for the even denominator $\mathrm{FQH}$ state have been proposed and discussed and it would seem, in general, as though its structure has not yet been clarified.

\section{Further Topics}

Space precludes a detailed account of further interesting topics so I shall only attempt to enumerate a few of the most recent developments. They involve experimental measurement techniques, notably optical detection methods [17], which complement transport measurements $[3$, 18 ] that are mainly sensitive to edge currents, and the investigation of sound propagation, which has recently given evidence for interesting anomalies at $v=1 / 2$ [19]. Finally, I should like to mention the evidence for the formation of an electron (Wigner) solid in the vicinity of the quantum Hall plateau at $v=1 / 5[20]$, and work on transitions between Hall plateaux for different $v$ where critical behaviour is found as $T \rightarrow 0 \mathrm{~K}$.

Concerning recent theoretical developments, work [21] concerned with the iden- tification of an order parameter and leading to Ginzburg-Landau type theories of the FQHE must be noted.

\section{Conclusions}

Observing the two-dimensional electron system in a high magnetic field has led to many fascinating discoveries. Some absolutely unexpected effects have been encountered, some of which, e.g. the even denominator fractional quantum Hall state at a Landau level filling fraction $v=5 / 2$, call for further analysis. Indeed, more than 10 years after its discovery, the quantum Hall effect remains full of new surprises.

\section{REFERENCES}

[1] Imry Y., Europhysics News 16 (1985) 1; The Quantum Hall Effect, Eds. R.E. Prange and S.M. Girvin (Springer, Berlin) 1990.

[2] Laughlin R.B., Phys. Rev. B 23 (1981) 5632.

[3] Halperin B.I., Phys. Rev. B 25 (1982) 2185; see also Imry Y., J. Phys. C 15 (1982) L221. In connection with the FQHE, the gauge invariance argument was recently discussed by Thouless D.J., Phys. Rev. B 40 (1989) 12034 and by Thouless D.J. and Gefen Y. Phys. Rev. Lett. 66 (1991) 806. [4] Tsui D.C., Störmer H.L. and Gossard A.C., Phys. Rev. Lett. 48 (1982) 1559.

[5] Laughlin R.B., Phys. Rev. Lett. 50 (1983) 1395.

[6] Haldane F.D.M., Phys. Rev. Lett. 51 (1983) 605; Yoshioka D.J., Phys. Rev. B 29 (1984) 6833.

[7] Haldane F.D.M. and Rezayi E.H., Phys. Rev. Lett. 54 (1985) 237.

[8] Willett R.L. et al., Phys. Rev. B 37 (1988) 8476.

[9] Halperin B.I., Phys. Rev. Lett. 52 (1984) 1583; ibid. 2390 (E). For a discussion of fractional statistics of quasiparticles, see also: Arovas D., Schrieffer J.R. and Wilczek F., Phys. Rev. Lett. 53 (1984) 722.

[10] d'Ambrumenil N. and Morf R., Phys. Rev. B 40 (1989) 6108. For a review of finite system calculations, see also: Chakraborty T. and Pietiläinen P., The Fractional Quantum Hall Effect (Springer, Berlin) 1988.

[11] Béran P. and Morf R., Phys. Rev. B 43 (1991) 12654.

[12] Clark R.G. et al., Phys. Rev. Lett. 60 (1988) 1747.

[13] Eisenstein J.P. et al., Phys. Rev. Lett. 62 (1989) 1540.

[14] Clark R.G. et al., Phys. Rev. Lett. 62 (1989) 1536.

[15] Willett R. et al., Phys. Rev. Lett. 59 (1987) 1776; Eisenstein J.P. et al., Phys. Rev. Lett. 61 (1988) 997.

[16] Haldane F.D.M. and Rezayi E.H., Phys. Rev. Lett. 60 (1988) 956; ibid. 1886.

[17] Turberfield A.J. et al., Phys. Rev. Lett. 65 (1990) 637; Goldberg B.B. et al., Phys Rev. Lett. 65 (1990) 641.

[18] Büttiker M., Phys. Rev. B 38 (1988) 9375.

[19] Willett R.L. et al., Phys. Rev. Lett. 65 (1990) 112.

[20] Jiang H.W. et al., Phys. Rev. Lett. 65 (1990) 633.

[21] Read N., Phys. Rev. Lett. 62 (1988) 86; Lee D.H. and Zhang S.C., Phys. Rev. Lett. 66 (1991) 1220 